\title{
PWM based Motor Speed Control using PIC Micro Controller
}

\author{
S. Nirmalraj ${ }^{1 *}$, V. Senthil Nayagam ${ }^{2}$, M. Kavitha ${ }^{3}$ Jayaprakash $^{4}$, A. Samhith ${ }^{5}$, A.Samhitha ${ }^{6}$ \\ \{nirmalraj.eeee@sathyabama.ac.in ${ }^{1}$, sensribala@gmail.com, kaveem@gmail.com\} \\ Assistant Professor ${ }^{1-4}$, Sathyabama Institute of Science and Technology, Chennai, India
}

\begin{abstract}
This paper is proposed DC motor for speed controlling using PWM control with the PIC microcontroller. The DC machine is explicitly comparing to the voltage associated over its terminals for controlling the speed. Consequently, on the off chance that the voltage over the terminal of motor is moved, at that point the speed can in like manner be changed. This venture uses the above rule to control the speed of the DC machine by fluctuating the duty cycle of the pulse associated with this. This task uses two data gets interfaced to the microcontroller, which are used to control the speed of motor.
\end{abstract}

Keywords: Pulse withModualtion (PWM), Direct currnet (DC) motor, Duty Cycle, Motor Driver Integrated Circuit (IC)

\section{Introduction}

The advancement has wiped out utilization of such huge hardware's, which were anticipated to offer approach to ac drives [1]. The paper gives a framework that can used to utilize DC motor for different applications [2]. The DC Motor is used for different applications by controlling the speed and direction as per the field of intrigue. Pulse Width Modulation (PWM) is the procedure of using switching devices to deliver the impact of a consistently fluctuating analog signal. This PWM change for the most part has very high electrical proficiency and can be utilized in controlling of drives (motor) [3]. The speed of the geared motors will be less with the increasing torque.Equipped motors generally comprise of a DC brush motor and a gearbox joined to the shaft.

\section{Proposed System Description}

The proposed method is expected to control the dc motor speed utilizing pwm control with the pic microcontroller[4].The dc motor speed will rely upon the voltage over the terminals. This paper uses the above principle to control dc motorspeed by altering the duty cycle associated with it(prevently known as pwm control) [5]. In this system speed will be displayed on LCD display. Here the AC current is used as power supply and step down transformer is used to convert $230 \mathrm{~V}$ into $12 \mathrm{~V}$ because in PIC microcontrollers only we can give $\mathrm{AC}$ current directly as power supply we cannot give $\mathrm{DC}$ current directly.To convert $\mathrm{AC}$ current to DC current rectifiers are used Those push buttons in which program is dumped by 
using device utilized to control the motor speed at various levels of duty cycle $(100 \%$ $, 80 \%, 60 \%, 40 \%$ ) duty cycles. Figure 2.1 shows the functional Representation of the proposed techniques.

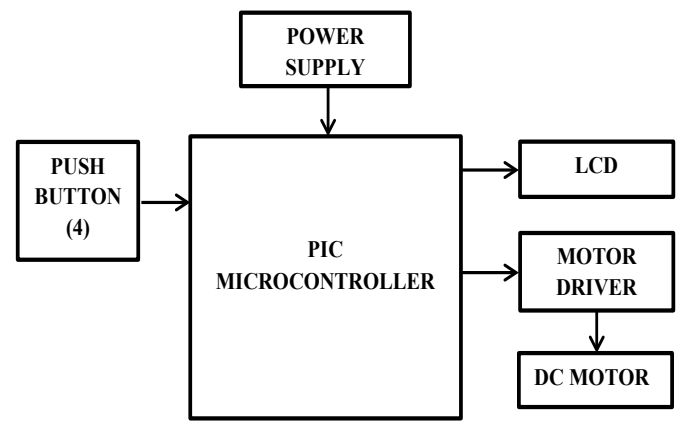

Figure 2.1 Functional Representation of the proposed techniques

The proposed framework presents the design of the speed control system for the DC Motor by utilizing PIC microcontroller.We have four switches customized to change the speed of motor. This framework would have the option to control the DC motor speed at the load change. By tweaking the pulse width enforced to the DC motor, the power applied to the motor can be changed which inturn controls the motor speed. Figure 2.2 represents the Circuit diagram of PWM based motor speed control using PIC microcontroller

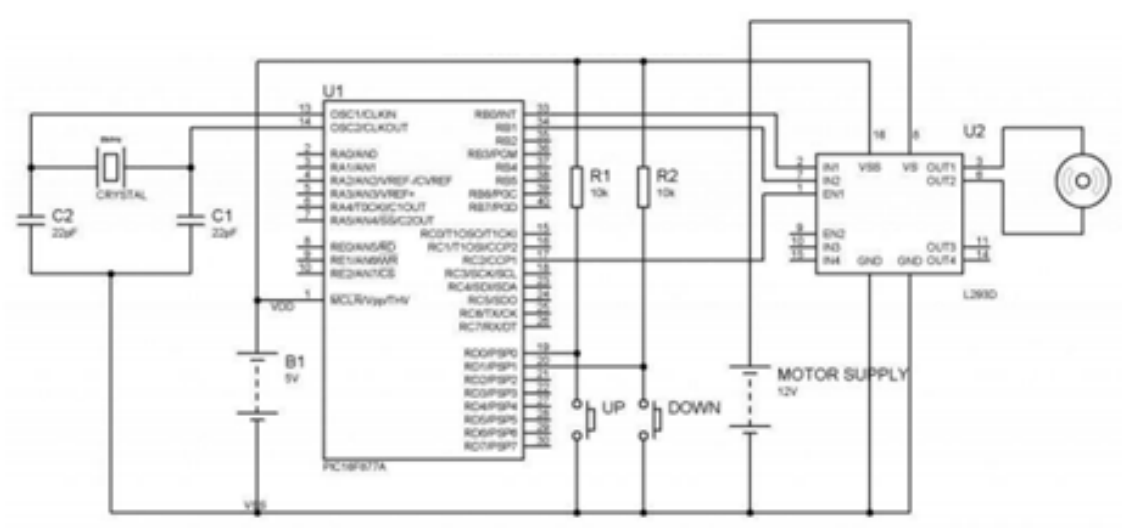

Figure 2.2 Circuit diagram of PWM based motor speed control using PIC microcontroller 


\section{Technical Specifications}

Pic Microcontroller: One of the prior forms of PIC Microcontrollers is PIC16C6x/7x. The $7 \mathrm{x}$ family has an upgrade of Analog to Digital converter converter. The PIC 16FXX utilizes Harvard design, in which, program and information are gotten to from discrete recollections utilizing separate bus. This improves transmission capacity over conventional Von Neumann design where program and information might be gotten from a similar memory utilizing a similar bus. Isolating program and data buts further permits instructions to be estimated uniquely in contrast to 8-cycle wide information words. Figure 3.1 shows the archietecture of PIC microcontroller. 10 bit ADC converterr is present in the PIC microcontroller which includes the amplifier circuit inside[6].

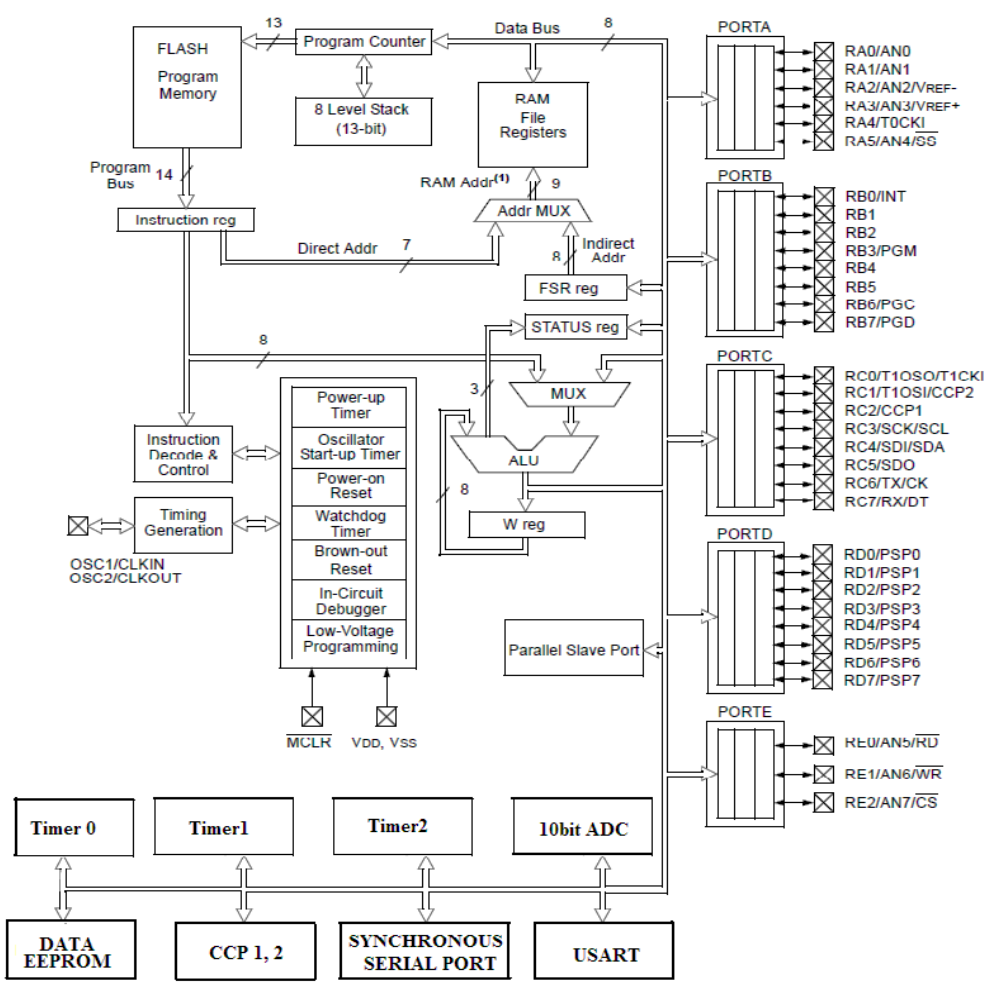

Figure 3.1 Archietecture of PIC microcontroller.

DC MOTOR. A DC motor that runs on DC power depends on the principle electromagnetism. A magnetic field is created by the conductor when a current flow through the conductor and it experiences a force when positioned in an external electric field. It is a device which changes electrical energy over to mechanical energy. It takes a shot at the way 
that a current carrying conductor set in a magnetic field experiences a force which makes it turn with deference to its original position. Figure 3.2 shows the DC motor connected to the motor control circuit [7],

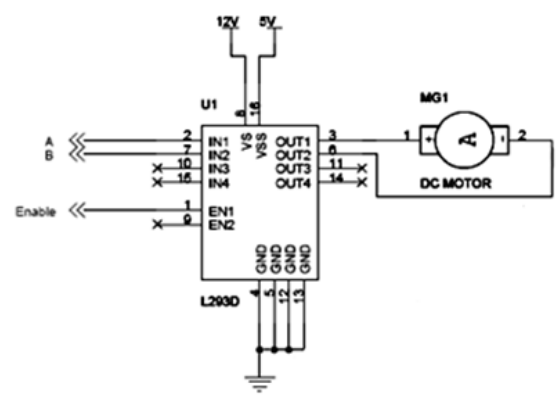

Figure 3.2 DC motor connected to H bridge motor control circuit L293D

Magnitude of flux experienced is,

$$
\text { FblI }
$$

The EMF induced in the opposite direction of the applied voltage is shown in equation (3.2)

$$
\begin{aligned}
& E b=\frac{\Phi P N Z}{60 A} \\
& T_{a} \frac{0.159 \Phi a P Z}{A}
\end{aligned}
$$

Thus the torque depends on the armature current. The change in the flow of armature current will change the speed of the motor.

Motor Driver IC: At the point when the motor is legitimately associated with the o/p then it may get harm. To beat this issue, there is a need of a motor control circuit. The different methods of making H-bridge motor control circuit, for example, utilizing transistors, relays and L293D/L29. A H connect circuit permits a voltage to be applied over a load toward any direction. H-connect circuits are as often as possible utilized in various applications to permit DC motors to run forward and in reverse.

A H-connect is manufactured with four switches like S1, S2, S3 and S4. A positive voltage is applied over the motor when the switches $\mathrm{S} 1$ and $\mathrm{S} 4$ are closed. The applied voltage is reversed when the switches S1 and S4 are open whereas the switches S2 and S3 are closed permitting reverse operation of the motor.

Toggle Switch:Here we are utilizing four customized push buttons which are toggle switches. Those push buttons in which program is loaded by utilizing devices used to control the speed of the engine at various levels of duty cycle. This push buttons are associated with thePIC microcontroller at one end all the push buttons are given to $5 \mathrm{~V}$ where as opposite end 
of the push buttons is given to the ground. Here program we utilized is embedded C. We need to on the individually push buttons to control the speed of the DC motor once one press button is on other ought to be off, So DC motor can be controlled at various rates.

\section{Simulation Results and Discussion}

We have utilized a PIC16F876 microcontroller to produce PWM and to change the duty cycle by perusing the simple estimation of voltage over the variable resistor. A $12 \mathrm{v}$ voltage source is utilized. A 7805 voltage controller is utilized to give 5 volts to the variable resistor and power supply to the microcontroller. A variable resistor is utilized to change the duty cycle of PWM. By moving the knob of a variable resistor the duty cycle can be changed which is legitimately identified with DC motor speed. In this way, DC motor speed can be changed by varying the variable resistor knob as indicated by your speed requirement. The PWM frequency is about $50 \mathrm{hz}$ which won't make any discernible noise. When Push button 1 is turned on the motor runs at full speed, when the push button 2 is turned on then the motor runs at $80 \%$ dutycycle. When Push button 3 and 4 are turned on the motor runs at $60 \%$ and $\$ 0 \%$ duty cycles respecrtively.

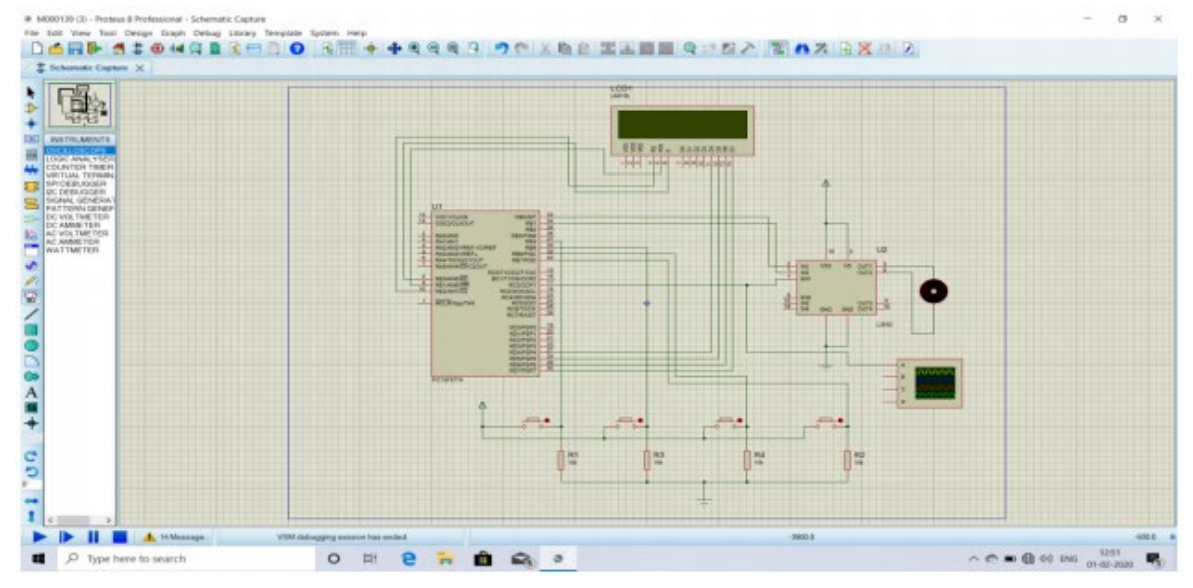

Figure 4.1 Simulation Circuit Diagram of PWM based Motor Speed Control Using Pic Microcontroller
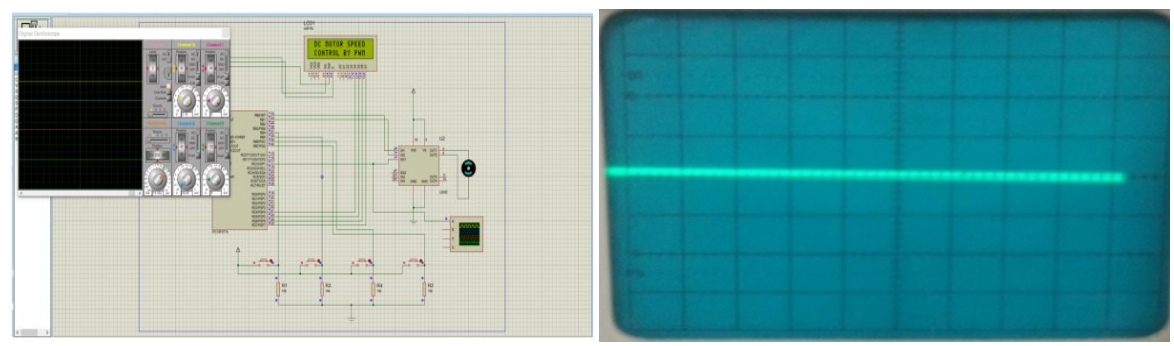

Figure 4.2 Motor runs at $100 \%$ duty cycle 


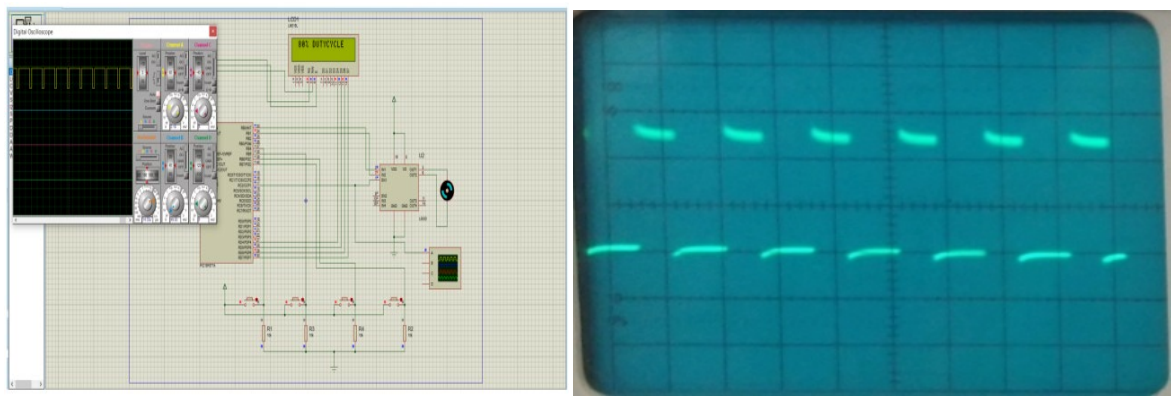

Figure 4.3 Motor runs at $80 \%$ duty cycle

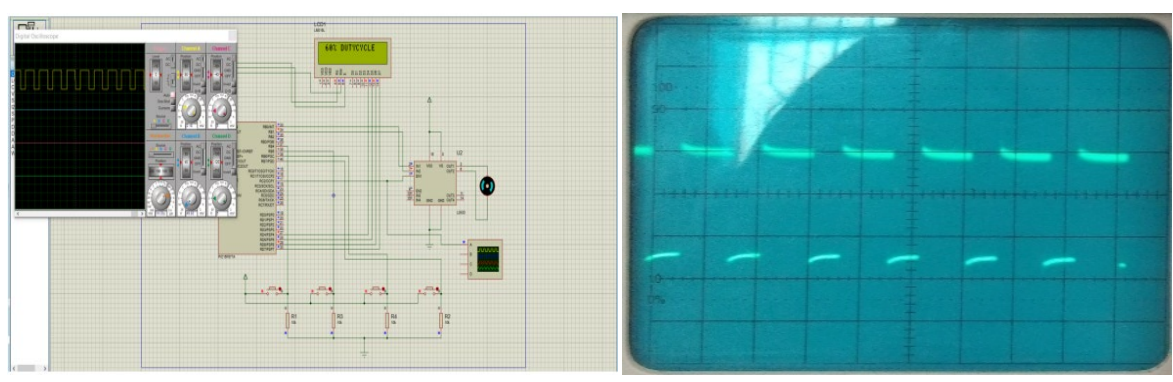

Figure 4.4 Motor runs at $60 \%$ duty cycle

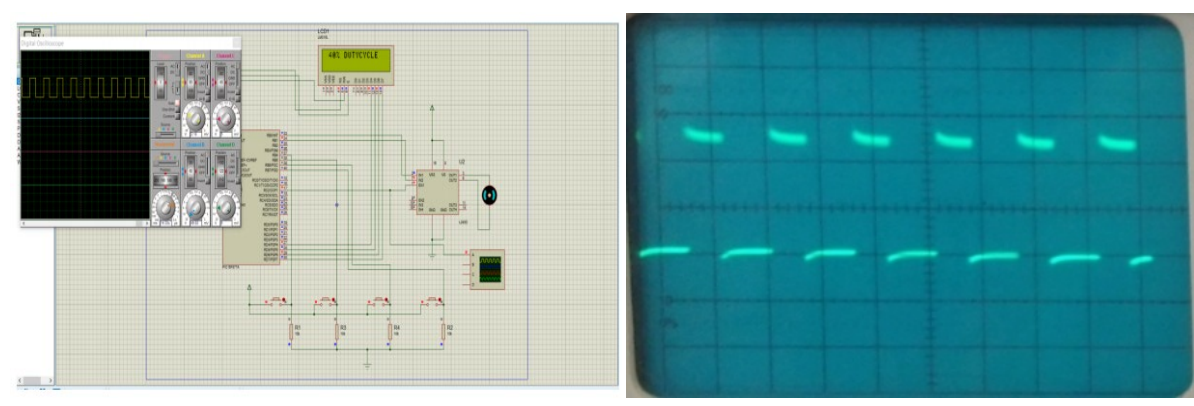

Figure 4.5 Motor runs at $100 \%$ duty cycle 


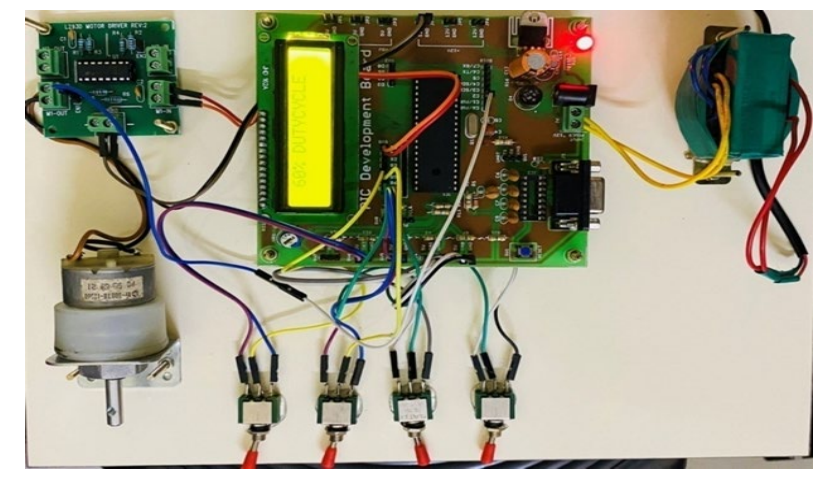

Figure 4.6 Harware model of the proposed system

\section{Conclusion}

This Venture is expected to control the DC motor speed using PWM control with the PIC microcontroller. The speed of the DC Engine will rely upon the voltage over the terminals of engine. By shifting the voltage over the terminals speed can be changed, this undertaking uses the above principle to control the speed of the DC engine by fluctuating the duty cycle of the pulse associated with it (predominantly known as PWM control). Additionally, the reenactment of proposed work has been done utilizing PROTEUS/SIMULINK and exploratory qualities are approved with simulation results. Results show that the speed of the DC motor can be changed effectively with less time utilized by the proposed venture. This circuit can be executed in house for viable utilization of water and electricity. 


\section{References}

[1] Aslam, S., Hannan, S., Haider, A., \& Tariq, M.H.Exploring PIC 24F"series Microcontroller using MPLAB and Proteus". (2016), Journal of Current Research in Science, 4(2), 164.

[2] Mishra, R., \& Elisabeth, E. A." Speed control of ceiling fan using PWM technique. In 2017 International Conference on Computation of Power, Energy Information and Commuincation "(ICCPEIC)(2017), (pp. 686-690). IEEE

[3] Vanitha Soman and Sudhakar S Mande, "Design of a Two-Stage Folded Cascode Amplifier Using SCL $180 \mathrm{~nm}$ CMOS Technology", International Conference on Communication, Computing and Electronics Systems Series, (ICCCES), (pp. 423-430), Springer,2020.

[4] Askar, P. A., Naphade, P. P., Jupaka, A. R., \&Adle, R. V." Speed Control of DC Motor Using Microcontroller". (2017), Journal of Network Communications and Emerging Technologies (JNCET), www. jncet. org, 7(3).

[5] Rex, S. R., \&Praba, M. S. R.” A Speed Control of DC Motor with PWM Using Microcontroller in Hardware in Loop".(2018), International Journal of Engineering \& Technology, (7(3.27), 116-119.

[6] Fatma, M. W., \& Hamid, M. I." PWM speed control of dc permanent magnet motor using a PIC18F4550 microcontroller". (2019), In IOP Conference Series: Materials Science and Engineering, IOP Publishing.

[7] P Illavarason, Renjith J Arokia, P Mohan Kumar, 2019, "Comparative study and an improved algorithm for iris and eye corner detection in real time application "Computer-Aided Developments: Electronics and Communication, CRC Press, PP-91-98. 